TRANSACTIONS OF THE

AMERICAN MATHEMATICAL SOCIETY

Volume 351, Number 5, Pages 1975-1987

S 0002-9947(99)02214-X

Article electronically published on January 27, 1999

\title{
GEOMETRY OF CANTOR SYSTEMS
}

\author{
YUNPING JIANG
}

\begin{abstract}
A Cantor system is defined. The geometry of a certain family of Cantor systems is studied. Such a family arises in dynamical systems as hyperbolicity is created. We prove that the bridge geometry of a Cantor system in such a family is uniformly bounded and that the gap geometry is regulated by the size of the leading gap.
\end{abstract}

\section{INTRODUCTION}

During the last two decades, Cantor sets have played an important role in the study of chaotic dynamical systems. One example is the structural stability theory developed by Smale [SM] and others; a second is the universality theory discovered by Feigenbaum [FE1], [FE2] (independently, by Coullet and Tresser [CT]), and developed by, among others, Lanford [LA1], [LA2], Sullivan [SU], and McMullen [MC]. In the second example, the geometry of Cantor sets presents a universal pattern. The study of the geometry of Cantor sets thus becomes an interesting and important problem.

In order to construct a Cantor set in the real line $\mathbb{R}$, we need to remove infinitely many subintervals which are called the gaps of the Cantor set. The sizes and positions of these gaps determine the geometry of the Cantor set. Let us first give a definition of an interval system which determines a Cantor set in the real line $\mathbb{R}$. Let $\mathcal{I}=\left\{\mathcal{I}_{n}\right\}_{n=0}^{\infty}$ be a sequence of families of disjoint, non-empty, compact intervals. Let $\mathcal{G}=\left\{\mathcal{G}_{n}\right\}_{n=1}^{\infty}$ be a sequence of families of disjoint, non-empty, open intervals. Let $\mathcal{C}=\{\mathcal{I}, \mathcal{G}\}$.

Definition 1. We call $\mathcal{C}$ a Cantor system if

(i) for each $0 \leq n<\infty$ and each interval $I \in \mathcal{I}_{n}$, there is a unique interval $G$ in $\mathcal{G}_{n+1}$ and two intervals $L$ and $R$ in $\mathcal{I}_{n+1}$ which lie to the left and to the right of $G$ such that $I=L \cup G \cup R$ (see Figure 1), and

(ii) $C S=\bigcap_{n=0}^{+\infty} \bigcup_{I \in \mathcal{I}_{n}} I$ is totally disconnected.

The set $C S$ in Definition 1 is a Cantor set in the real line $\mathbb{R}$. We call each interval $I$ in $\mathcal{I}_{n}$ for $0 \leq n<\infty$ a $\mathcal{C}$-bridge and call each interval $G$ in $\mathcal{G}_{n}$ for $1 \leq n<\infty$ a $\mathcal{C}$-gap. We also call each interval $G$ in $\mathcal{G}_{1}$ a leading gap. We note that a Cantor system determines a unique Cantor set in the real line $\mathbb{R}$.

Definition 2. The bridge geometry of $\mathcal{C}$ is the set of ratios

$\mathcal{B R}=\left\{\frac{|J|}{|I|} ; I=L \cup G \cup R \in \mathcal{I}_{n}, J=L\right.$ or $\left.R \in \mathcal{I}_{n+1}, G \in \mathcal{G}_{n+1}, n=0,1,2, \ldots\right\}$.

Received by the editors February 12, 1996 and, in revised form, December 2, 1996.

1991 Mathematics Subject Classification. Primary 57F25, 58F11.

Partially supported by an NSF grant and PSC-CUNY awards. 


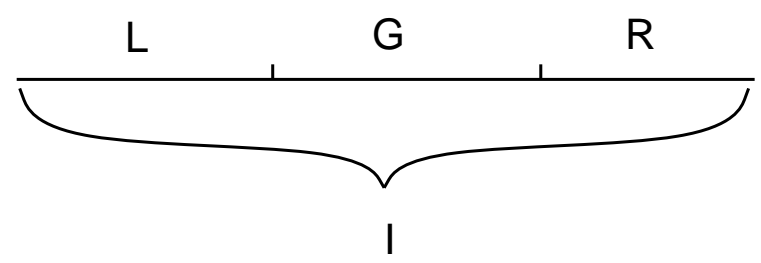

Figure 1

The gap geometry of $\mathcal{C}$ is the set of ratios

$\mathcal{G} \mathcal{A} \mathcal{P}=\left\{\frac{|G|}{|J|} ; I=L \cup G \cup R \in \mathcal{I}_{n}, J=L\right.$ or $\left.R \in \mathcal{I}_{n+1}, G \in \mathcal{G}_{n+1}, n=0,1,2, \ldots\right\}$.

In this paper, we study the bridge geometry and the gap geometry of a family of Cantor systems which depend on $\epsilon$. Such a family arises in dynamical systems as hyperbolicity is created. One example of such a family is the family of Cantor systems $\left\{\mathcal{C}_{\epsilon}\right\}_{0<\epsilon \leq 1}$ dynamically defined by the family of self-maps $\left\{f_{\epsilon}(x)=1+\epsilon-\right.$ $\left.(2+\epsilon)|x|^{\gamma}\right\}$ of the interval $[-1,1]$, where $\gamma>1$ is fixed and $\epsilon>0$ is a parameter. Another example is the family of Cantor systems $\left\{\tilde{\mathcal{C}}_{\epsilon}\right\}_{0<\epsilon \leq 1}$ dynamically defined by the family of self-maps $\left\{g_{\epsilon}(x)=-1+(2+\epsilon)(\cos (\pi x / 2))^{\gamma / 2}\right\}$ of the interval $[-1,1]$, where $\gamma>1$ is fixed and $\epsilon>0$ is a parameter. In these examples, the dynamical systems generated by $f_{\epsilon}$ and $g_{\epsilon}$ are hyperbolic when $\epsilon>0$ and cease to be hyperbolic when $\epsilon=0$. The corresponding Cantor sets are the maximal invariant sets of $f_{\epsilon}$ and $g_{\epsilon}$. One of the main results in this paper shows that in a family of Cantor systems like these two examples, the bridge geometry is bounded uniformly and the gap geometry is regulated by the function $\alpha: \epsilon \mapsto \epsilon^{\frac{1}{\gamma}}$.

The paper is organized as follows. In $\S 2$, we define an asymptotically nonhyperbolic family of folding mappings. In $\S 3$, we study families of linear Cantor systems depending on parameters $\epsilon$. We find some conditions on families of linear Cantor sets depending on parameters $\epsilon$ such that the gap geometry is regulated by the function $\alpha(\epsilon)=\epsilon^{\frac{1}{\gamma}}$, and, for each $\epsilon>0$, the deviation of the Hausdorff dimension of the corresponding Cantor set from one is comparable to $\epsilon^{\frac{1}{\gamma}}$. This is formulated as Theorem 2 and gives us some insight into why the study of nonlinear Cantor systems depending on parameters $\epsilon$ is interesting and important. In $\S 5$, we study a family of interval systems $\left\{\mathcal{C}_{\epsilon}\right\}_{0 \leq \epsilon \leq \epsilon_{0}}$ dynamically defined by an asymptotically non-hyperbolic family of non-linear folding mappings $\left\{f_{\epsilon}\right\}_{0 \leq \epsilon \leq \epsilon_{0}}$. Let $\mathcal{B R}_{\epsilon}$ be the bridge geometry and $\mathcal{G} \mathcal{A} \mathcal{P}_{\epsilon}$ be the gap geometry of $\mathcal{C}_{\epsilon}$ for each $0<\epsilon \leq \epsilon_{0}$. Let $\alpha(\epsilon)>0$ be a function of $\epsilon$. We prove in $\S 5$ one of our main results:

Theorem 1. For each $0<\epsilon \leq \epsilon_{0}$, the dynamically defined interval system $\mathcal{C}_{\epsilon}$ is a Cantor system whose bridge geometry is uniformly bounded and whose gap geometry is regulated by the function $\alpha: \epsilon \mapsto \epsilon^{\frac{1}{\gamma}}$; more precisely, there is a constant $K>0$ such that

$$
|\log (b r(\epsilon))| \leq K
$$

and

$$
\left|\log \left(\frac{g g(\epsilon)}{\alpha(\epsilon)}\right)\right| \leq K
$$


for all $\operatorname{br}(\epsilon) \in \mathcal{B R}_{\epsilon}$ and all $g g(\epsilon) \in \mathcal{G} \mathcal{A} \mathcal{P}_{\epsilon}$ and all $0<\epsilon \leq \epsilon_{0}$.

Controlling the nonlinearity of the dynamical system generated by $f_{\epsilon}$ is one of main themes in the proof. In $\S 4$, we review some properties of the Schwarzian derivatives. A result about controlling the nonlinearity of a $C^{3}$ diffeomorphism by using its Schwarzian derivative is also proved in this section. A corollary of this result is the $C^{3}$ Koebe distortion lemma (see [CE], [MV]). The main idea involved in the proof of Theorem 1 is to divide the interval where the function $f_{\epsilon}$ is defined into two parts. The first part is away from the critical point of $f_{\epsilon}$. The second part is away from the post-critical orbit of $f_{\epsilon}$. In the first part, we use the naive distortion property (see Lemma 1) to control the nonlinearity of the dynamical system generated by $f_{\epsilon}$. Theorem 3 (see Lemma 2) takes care of the nonlinearity in the second part. The power law function $x \mapsto-|x|^{\gamma}$ makes the transition from the first part into the second part.

Acknowledgment. The author would like to thank Professor Dennis Sullivan for insightful suggestions.

\section{An ASYMPTOTICALLY NON-HYPERBOLIC FAMILY}

Let $[a, b]$ be the closed interval bounded by $a$ and $b$ and let $\epsilon_{0}>0$ and $\gamma>1$ be real numbers. The Schwarzian derivative $S(h)$ of a $C^{3}$-diffeomorphism $h$ from an interval $I$ onto $h(I)$ is, by definition,

$$
S(h)=\frac{h^{\prime \prime \prime}}{h^{\prime}}-\frac{3}{2}\left(\frac{h^{\prime \prime}}{h^{\prime}}\right)^{2} .
$$

Let $\mathcal{F}=\left\{f_{\varepsilon}\right\}_{0 \leq \epsilon \leq \epsilon_{0}}$ be a family of folding mappings.

Definition 3. We say $\mathcal{F}$ is asymptotically non-hyperbolic if

(a) every $f_{\epsilon}(x)=h_{\epsilon}\left(-|x|^{\gamma}\right)$ where $h_{\epsilon}:[-1,0] \rightarrow[-1,1+\epsilon]$ is a $C^{3}$ orientationpreserving diffeomorphism such that $S\left(h_{\epsilon}\right)(x) \leq 0$ for all $x$ in $[-1,0]$ and such that $h_{\epsilon}(-1)=-1$ and $h_{\epsilon}(0)=1+\epsilon$ for all $x$ in $[-1,0]$ and all $0 \leq \epsilon \leq 1$,

(b) there is a constant $K>0$ such that $\left(\log h_{\epsilon}^{\prime}(x)\right)^{\prime} \leq K$ for all $-1 \leq x \leq 0$ and all $0 \leq \epsilon \leq \epsilon_{0}$, and

(c) there is a constant $\lambda>1$ such that $f_{\epsilon}^{\prime}(-1) \geq \lambda$ for all $0 \leq \epsilon \leq \epsilon_{0}$.

Let $\mathcal{F}$ be an asymptotically non-hyperbolic family. For each $0<\epsilon \leq \epsilon_{0}$, let $\mathcal{I}_{n, \epsilon}$ be the set of intervals in $f_{\epsilon}^{-n}([-1,1])$ and let $\mathcal{G}_{n, \epsilon}$ be the set of intervals in $f_{\epsilon}^{-n}((1,1+\epsilon))$. Let $\mathcal{I}_{\epsilon}=\left\{\mathcal{I}_{n, \epsilon}\right\}_{n=0}^{\infty}$, let $\mathcal{G}_{\epsilon}=\left\{\mathcal{G}_{n, \epsilon}\right\}_{n=1}^{\infty}$, and let $\mathcal{C}_{\epsilon}=\left\{\mathcal{I}_{\epsilon}, \mathcal{G}_{\epsilon}\right\}$. Then $\mathcal{C}_{\epsilon}$ is an interval system dynamically defined by $f_{\epsilon}$. For $0<\epsilon \leq \epsilon_{0}$, the leading gap of $\mathcal{C}_{\epsilon}$ is $G_{*, \epsilon}=f_{\epsilon}^{-1}((1,1+\epsilon))$ and its size $\lg (\epsilon)$ is

$$
l g(\epsilon)=\left(\frac{\epsilon}{h_{\epsilon}^{\prime}(\xi)}\right)^{\frac{1}{\gamma}}
$$

for some $\xi \in[-1,0]$. From (b) of Definition 3 and the fact that $h_{\epsilon}([-1,0])=$ $[-1,1+\epsilon]$, there is a constant $K>0$ independent of $\epsilon$ such that

$$
\left|\log h_{\epsilon}^{\prime}(x)\right| \leq K
$$


for all $-1 \leq x \leq 0$ and all $0 \leq \epsilon \leq \epsilon_{0}$. Thus there is a constant $K>0$ independent of $\epsilon$ such that

$$
\left|\log \left(\frac{\lg (\epsilon)}{\epsilon^{\frac{1}{\gamma}}}\right)\right| \leq K
$$

for all $0<\epsilon \leq \epsilon_{0}$. The following theorem says that this inequality is true not only for the leading gaps but also for all gaps. Let $\mathcal{B R}_{\epsilon}$ be the bridge geometry and $\mathcal{G} \mathcal{A} \mathcal{P}_{\epsilon}$ be the gap geometry of $\mathcal{C}_{\epsilon}$.

Theorem 1. For each $0<\epsilon \leq \epsilon_{0}$, the dynamically defined interval system $\mathcal{C}_{\epsilon}$ is a Cantor system whose bridge geometry is uniformly bounded and whose gap geometry is regulated by the function $\alpha: \epsilon \mapsto \epsilon^{\frac{1}{\gamma}}$; more precisely, there is a constant $K>0$ such that

$$
|\log (b r(\epsilon))| \leq K
$$

and

$$
\left|\log \left(\frac{g g(\epsilon)}{\alpha(\epsilon)}\right)\right| \leq K
$$

for all br $(\epsilon) \in \mathcal{B R}_{\epsilon}$ and all $g g(\epsilon) \in \mathcal{G} \mathcal{A} \mathcal{P}_{\epsilon}$ and all $0<\epsilon \leq \epsilon_{0}$.

The proof of this theorem is given in $\S 5$. One of the consequences of Theorem 1 is that

$$
C S_{\epsilon}=\bigcap_{n=0}^{\infty} \bigcup_{I \in \mathcal{I}_{n, \epsilon}} I
$$

is a Cantor set on the real line $\mathbb{R}$ for each $0<\epsilon \leq \epsilon_{0}$.

Remark 1. For $\epsilon=0$, let $\mathcal{I}_{n, 0}$ be the set of the closures of intervals in $f_{0}^{-n}([-1,1))$. Let $\mathcal{I}_{0}=\left\{\mathcal{I}_{n, 0}\right\}_{n=0}^{\infty}$ and let $\mathcal{C}_{0}=\left\{\mathcal{I}_{0}, \emptyset\right\}$. Then $\mathcal{C}_{0}$ is an interval system dynamically defined by $f_{0}$. Although $C S_{0}=\bigcap_{n=0}^{\infty} \bigcup_{I \in \mathcal{I}_{n, 0}} I=[-1,1]$ is not a Cantor set, but from the dynamical system point of view, the interval system $\mathcal{C}_{0}$ dynamically defined by $f_{0}$ can be also considered as a Cantor system with null gaps and with bridges $\mathcal{I}_{0}=\left\{\mathcal{I}_{n, 0}\right\}_{n=0}^{\infty}$. Therefore $\mathcal{C}_{0}$ can be included in the first statement of Theorem 1 too.

Remark 2. Let $\tilde{\mathcal{F}}=\left\{\tilde{f}_{\epsilon}\right\}_{0 \leq \epsilon \leq \epsilon_{0}}$ be another asymptotically non-hyperbolic family of folding mappings and let $\mathcal{H}=\left\{H_{\epsilon}\right\}_{0 \leq \epsilon \leq \epsilon_{0}}$ be a family of homeomorphisms of $[-1,1]$ such that

$$
\tilde{f}_{\epsilon} \circ H_{\epsilon}\left|C S_{\epsilon}=H_{\epsilon} \circ f_{\epsilon}\right| C S_{\epsilon} .
$$

From Theorem 1, we can also prove that $H_{\epsilon} \mid C_{\epsilon}$ is quasisymmetric (see $[\mathrm{AH}]$ ). In particular, $H_{0}$ is a quasisymmetric homeomorphism of $[-1,1]$. This result has been generalized to geometrically finite one-dimensional maps. The proof of the generalized result can be found in [JI1].

Remark 3. From Theorem 1, one can see that the Hausdorff dimension of $H D(\epsilon)$ of $C_{\epsilon}$ is less than or equal to $1-K \epsilon^{\frac{1}{\gamma}}$, where $K>0$ is a constant (see [JI2] for some estimate of $H D(\epsilon)$ from below). 


\section{CANTOR SYSTEMS GENERATED By PIECEWISE LINEAR MAPPINGS}

In this section, we discuss some examples of families of Cantor systems dynamically defined by piecewise linear mappings. The discussion gives us some insight into why Theorem 1 is interesting and important.

Let $l=l(\epsilon)$ and $r=r(\epsilon)$ be two positive functions on $\left(0, \epsilon_{0}\right]$ satisfying

$$
l(\epsilon)+r(\epsilon)<1
$$

Define

$$
f_{\epsilon}(x)= \begin{cases}\frac{x}{l(\epsilon)} & \text { for } x \in[0, l(\epsilon)] \\ \frac{1-x}{r(\epsilon)} & \text { for } x \in[1-r(\epsilon), 1] .\end{cases}
$$

For each $0<\epsilon \leq \epsilon_{0}$ and for each $0 \leq n<\infty$, let $\mathcal{I}_{n, \epsilon}$ be the set of intervals in $f_{\epsilon}^{-n}([0,1])$. Let $G_{*, \epsilon}=(l(\epsilon), 1-r(\epsilon))$ and let $\mathcal{G}_{1, \epsilon}=\left\{G_{*, \epsilon}\right\}$. Let $\mathcal{G}_{n, \epsilon}$ be the set of intervals in $f_{\epsilon}^{-n+1}\left(G_{*, \epsilon}\right)$ for all $2 \leq n<\infty$. Let $\mathcal{I}_{\epsilon}=\left\{\mathcal{I}_{n, \epsilon}\right\}_{n=0}^{\infty}$, let $\mathcal{G}_{\epsilon}=\left\{\mathcal{G}_{n, \epsilon}\right\}_{n=1}^{\infty}$, and let $\mathcal{L} C_{\epsilon}=\left\{\mathcal{I}_{\epsilon}, \mathcal{G}_{\epsilon}\right\}$. The size of the leading gap $G_{*, \epsilon}$ is $l g(\epsilon)=1-l(\epsilon)-r(\epsilon)$.

Definition 4. We call $\left\{\mathcal{L} C_{\varepsilon}\right\}_{0<\epsilon \leq \epsilon_{0}}$ a family of linear Cantor systems with $\epsilon^{\frac{1}{\gamma}}$ leading gaps if there is a constant $K>0$ such that

$$
\left|\log \left(\frac{\lg (\epsilon)}{\epsilon^{\frac{1}{\gamma}}}\right)\right| \leq K
$$

for all $0<\epsilon \leq \epsilon_{0}$, where $\gamma>1$ is a real number.

Let $\left\{\mathcal{L} C_{\varepsilon}\right\}_{0<\epsilon \leq \epsilon_{0}}$ be a family of linear Cantor systems with $\epsilon^{\frac{1}{\gamma}}$ leading gaps. Let $L C_{\epsilon}=\bigcap_{n=0}^{\infty} \bigcup_{I \in \mathcal{I}_{n, \epsilon}} I$. It is a Cantor set on the real line $\mathbb{R}$. Let $H D(\epsilon)$ be the Hausdorff dimension of $L C_{\epsilon}$. It is easy to see that there is a constant $K>0$ such that

$$
H D(\epsilon) \leq 1-K \epsilon^{\frac{1}{\gamma}}
$$

for all $0<\epsilon \leq \epsilon_{0}$. We give some sufficient conditions such that $1-H D(\epsilon)$ is comparable with $\epsilon^{\frac{1}{\gamma}}$.

Condition 1. Both $l(\epsilon)$ and $r(\epsilon)$ are continuous on $\left[0, \epsilon_{0}\right]$ and $l(0)$ and $r(0)$ are in $(0,1)$.

Condition 2. Both $l(\epsilon)$ and $r(\epsilon)$ are differentiable at every $0<\epsilon \leq \epsilon_{0}$ and there is a constant $K>0$ such that

$$
K^{-1} \leq-l^{\prime}(\epsilon) \epsilon^{\frac{\gamma-1}{\gamma}} \leq K \quad \text { and } \quad K^{-1} \leq-r^{\prime}(\epsilon) \epsilon^{\frac{\gamma-1}{\gamma}} \leq K
$$

for all $0<\epsilon \leq \epsilon_{0}$.

Theorem 2. Let $\left\{\mathcal{L} C_{\epsilon}\right\}_{0<\epsilon \leq \epsilon_{0}}$ be a family of linear Cantor systems with $\epsilon^{\frac{1}{\gamma}}$ leading gaps. If $l(\epsilon)$ and $r(\epsilon)$ satisfy Condition 1, then the gap geometry of $\left\{\mathcal{L} C_{\epsilon}\right\}_{0<\epsilon \leq \epsilon_{0}}$ is regulated by the function $\alpha: \epsilon \mapsto \epsilon^{\frac{1}{\gamma}}$ (refer to Theorem 1). Furthermore, if $l(\epsilon)$ and $r(\epsilon)$ satisfy both Conditions 1 and 2, then there is a constant $K>0$ independent of $\epsilon$ such that

$$
K^{-1} \epsilon^{\frac{1}{\gamma}} \leq 1-H D(\epsilon) \leq K \epsilon^{\frac{1}{\gamma}}
$$

for all $0<\epsilon \leq \epsilon_{0}$. 
Proof. The proof of the first statement is easy. We prove the second. Consider the equation

$$
(l(\epsilon))^{H D(\epsilon)}+(r(\epsilon))^{H D(\epsilon)}=1 .
$$

The implicit function theorem tells us that $H D(\epsilon)$ is differentiable at every $\epsilon, 0<$ $\epsilon \leq \epsilon_{0}$. With some calculation, we have

$$
(\log (H D(\epsilon)))^{\prime}=-\frac{(l(\epsilon))^{H D(\epsilon)-1} l^{\prime}(\epsilon)+(r(\epsilon))^{H D(\epsilon)-1} r^{\prime}(\epsilon)}{(l(\epsilon))^{H D(\epsilon)} \log (l(\epsilon))+(r(\epsilon))^{H D(\epsilon)} \log (r(\epsilon))} .
$$

Therefore $-(\log (H D(\epsilon)))^{\prime}$ is comparable with $\epsilon^{\frac{1}{\gamma}-1}$. Thus there is a constant $K>0$ independent of $\epsilon$ such that

$$
K^{-1} \epsilon^{\frac{1}{\gamma}} \leq-\log (H D(\epsilon)) \leq K \epsilon^{\frac{1}{\gamma}}
$$

for all $0<\epsilon \leq \epsilon_{0}$. This implies the second statement.

\section{Properties of the Schwarzian Derivatives}

We first review some properties of the Schwarzian derivatives. We will not give the proofs for them since they are quite well-known. The reader may refer to [CE], $[\mathrm{MV}]$.

Let $\mathcal{D}^{3}$ be the space of $C^{3}$-diffeomorphisms $f: I \rightarrow J$ where $I$ and $J$ are intervals of the real line $\mathbb{R}$. The Schwarzian derivative $S(f)$ of $f$ in $\mathcal{D}^{3}$ is, by definition,

$$
S(f)=\frac{f^{\prime \prime \prime}}{f^{\prime}}-\frac{3}{2}\left(\frac{f^{\prime \prime}}{f^{\prime}}\right)^{2} \text {. }
$$

We use $S(f) \geq 0$ (or $S(f) \leq 0)$ to mean that $S(f)(x) \geq 0$ (or $S(f)(x) \leq 0)$ for all $x$ in $I$. We have the chain rule for the Schwarzian derivatives: for any two maps $g: K \rightarrow I$ and $f: I \rightarrow J$ in $\mathcal{D}^{3}$,

$$
S(f \circ g)=\left(g^{\prime}\right)^{2} \cdot S(f) \circ g+S(g) .
$$

The chain rule implies that $S(f \circ g) \geq 0$ and $S\left(f^{-1}\right) \leq 0$ if $S(f) \geq 0$ and $S(g) \geq 0$ and that $S(f \circ g) \leq 0$ and $S\left(f^{-1}\right) \geq 0$ if $S(f) \leq 0$ and $S(g) \leq 0$.

Let $\gamma>1$. It is easy to check that for every power function $P(x)=-|x|^{\gamma}: I \rightarrow J$ in $\mathcal{D}^{3}, S(P) \leq 0$; for every root function $R(x)=-|x|^{\frac{1}{\gamma}}: I \rightarrow J$ in $\mathcal{D}^{3}, S(R) \geq 0$; for every Möbius transformation $H(x)=(a x+b) /(c x+d): I \rightarrow J$ in $\mathcal{D}^{3}, S(H)=0$.

Let $T=[a, d]$ be an interval and let $M=[b, c]$ be a subinterval of $T$ where $a<b<c<d$. Let $L=[a, b]$ and $R=[c, d]$ be the closures of two connected components of $T \backslash M$. Let $|I|$ mean the length of an interval $I$. The Poincaré length $P_{T}(M)$ of $M$ in $T$ is defined as

$$
P_{T}(M)=\log \left(1+\frac{|T| \cdot|M|}{|L| \cdot|R|}\right) .
$$

For any $f: I \rightarrow I$ in $\mathcal{D}^{3}$ having $S(f) \geq 0$, then

$$
P_{f(T)}(f(M)) \leq P_{T}(M)
$$


for any $M \subset T \subseteq I$. This implies that

$$
\left|f^{\prime}(x)\right| \cdot \frac{|f(T)|}{|T|} \leq \frac{|f(R)|}{|R|} \cdot \frac{|f(L)|}{|L|}
$$

for any $f: I \rightarrow I$ in $\mathcal{D}^{3}$ having $S(f) \geq 0$ and any $M=\{x\} \subset T \subseteq I$.

Suppose that $f: I=[a, b] \rightarrow J$ is a map in $\mathcal{D}^{3}$ and that $S(f) \geq 0$. Then $g=1 / \sqrt{\left|f^{\prime}\right|}$ is a concave downward function on $I$ because $g^{\prime \prime}=-(1 / 2) g S(f)$. This implies that

$$
\left|f^{\prime}(x)\right| \leq \max \left\{\left|f^{\prime}(a)\right|,\left|f^{\prime}(b)\right|\right\}
$$

for any $x$ in $I$.

The nonlinearity $N(f)$ of $f$ in $\mathcal{D}^{3}$ is, by definition,

$$
N(f)=\frac{f^{\prime \prime}}{f^{\prime}}
$$

Controlling the nonlinearity of $f$ is an important topic in dynamical systems. It is easy to see that

$$
S(f)=(N(f))^{\prime}-\frac{1}{2}(N(f))^{2} .
$$

The next result shows how to control the nonlinearity of a map in $\mathcal{D}^{3}$ by using its Schwarzian derivative.

Theorem 3. Let $f: I=[a, b] \rightarrow J$ be a map in $\mathcal{D}^{3}$. Suppose there is a constant $K \geq 0$ such that $S(f)(x) \geq-K$ for all $x$ in $I$. Then, for any $\epsilon>0$,

$$
|N(f)(x)| \leq \max \left\{\sqrt{2 K+\epsilon}, \frac{2 K+\epsilon}{\epsilon} \frac{2}{d(x, \partial I)}\right\}
$$

for any $x$ in $I$, where $d(x, \partial I)=\min \{|x-a|,|x-b|\}$.

Proof. Let $x$ be any point in $I$. If $|N(f)(x)| \leq \sqrt{2 K+\epsilon}$, we have nothing to prove. Suppose $|N(f)(x)|>\sqrt{2 K+\epsilon}$ and suppose $N(f)(x)>0$ (the argument is similar when $N(f)(x)<0)$. For any $0 \leq t<b-x$, if $N(f)(x+t)>\sqrt{2 K+\epsilon}$, then

$$
N^{\prime}(f)(x+t)=\frac{1}{2}(N(f)(x+t))^{2}+S(f)(x+t) \geq \frac{\epsilon}{2}>0 .
$$

This implies that $N(f)(x+t)$ is a strictly increasing function of $t, t \in[0, b-x)$. Therefore, $N(f)(x+t) \geq \sqrt{2 K+\epsilon}$ for all $t \in[0, b-x)$.

Consider the ordinary differential equation,

$$
\left\{\begin{array}{l}
y^{\prime}(t)=\frac{1}{2} \frac{\epsilon}{2 K+\epsilon}(y(t))^{2} \\
y(0)=N(f)(x)
\end{array}\right.
$$

for $t$ in $[0, b-x)$. The unique solution of this equation is the function

$$
y_{0}(t)=\frac{1}{-\frac{1}{2} \frac{\epsilon}{2 K+\epsilon} t+\frac{1}{N(f)(x)}}
$$

for $t \in[0, b-x)$. Let

$$
t_{1}=\frac{2 K+\epsilon}{\epsilon} \frac{2}{N(f)(x)}
$$


be the unique pole of this solution. The function $y_{1}(t)=N(f)(t+x)$ on $[0, b-x)$ is a solution of the ordinary differential inequality

$$
\left\{\begin{array}{l}
y^{\prime}(t) \geq \frac{1}{2} \frac{\epsilon}{2 K+\epsilon}(y(t))^{2} \\
y(0)=N(f)(x)
\end{array}\right.
$$

for $t \in[0, b-x)$ since $S(f)(x+t) \geq-K$ and $N(f)(x+t) \geq \sqrt{2 K+\epsilon}$ for $t \in$ $[0, b-x)$. Then $y_{1}(t)$ is greater than or equal to $y_{0}(t)$ for $t \in[0, b-x)$ because of the comparison theorem for ordinary differential equations (see $[\mathrm{AR}]$ ). This implies that $y_{0}(t)$ is continuous on $[0, b-x)$. So the pole $t_{1}$ is greater than or equal to $b-x$. Hence

$$
N(f)(x) \leq \frac{2 K+\epsilon}{\epsilon} \frac{2}{b-x}
$$

which is less than or equal to $((2 K+\epsilon) / \epsilon)(2 / d(x, \partial I))$.

Corollary 1 ( $C^{3}$-Koebe distortion lemma). Suppose $f: I=[a, b] \rightarrow J$ is a map in $\mathcal{D}^{3}$ having $S(f)(x) \geq 0$ for all $x$ in $I$. Then

$$
|N(f)(x)| \leq \frac{2}{d(x, \partial I)}
$$

for any $x$ in $I$

\section{Cantor systems generated by NON-Linear mappingS}

We study a family of non-linear Cantor systems generated by a family of an asymptotically non-hyperbolic family of folding mappings and prove Theorem 1.

We use $K>0$ to denote a constant (even though it may be different in different formulas). Let $\mathcal{F}=\left\{f_{\epsilon}\right\}_{0 \leq \epsilon \leq \epsilon_{0}}$ be an asymptotically non-hyperbolic family of folding mappings. For each $0 \leq \epsilon \leq \epsilon_{0}$, let $f_{0, \epsilon}=f_{\epsilon} \mid[-1,0]$ and let $f_{1, \epsilon}=f_{\epsilon} \mid[0,1]$. We will omit $\epsilon$ if there can be no confusion.

Let $g_{0}:[-1,1+\epsilon] \rightarrow[-1,0]$ and $g_{1}:[-1,1+\epsilon] \rightarrow[0,1]$ be the inverses of $f_{0, \epsilon}$ and $f_{1, \epsilon}$, respectively. Let $w_{n}=i_{0} \ldots i_{n-1}$ denote a sequence of symbols 0 's and 1 's of length $n$ and let

$$
g_{w_{n}}=g_{i_{0}} \circ \cdots \circ g_{i_{n-1}}
$$

Let

$$
I_{w_{n}}=g_{w_{n}}([-1,1])
$$

Then

$$
\mathcal{I}_{n}=\left\{I_{w_{n}} \mid w_{n}=i_{0} \ldots i_{n-1} \text { is a sequence of 0's and 1's of length } n\right\}
$$

for $n=1,2, \ldots$ Let $G_{*}=[-1,1] \backslash\left(I_{0} \cup I_{1}\right)$ be the leading gap and let

$$
G_{w_{n}}=g_{w_{n}}\left(G_{*}\right) \text {. }
$$

Then $G_{w_{n}}$ is an open interval in $I_{w_{n}}$,

$$
\mathcal{G}_{n+1}=\left\{G_{w_{n}} \mid w_{n}=i_{0} \ldots i_{n-1} \text { is a sequence of 0's and 1's of length } n\right\}
$$

for $n=1, \ldots$, and $\mathcal{G}_{1}=\left\{G_{*}\right\}$.

Let $\left\{-a_{\epsilon}, a_{\epsilon}\right\}=f_{\epsilon}^{-1}(0)$ where $a_{\epsilon}>0$. From (b) of Definition 3 and the argument after Definition 3, there is a constant $0<K<1$ such that $K \leq a_{\epsilon} \leq 1-K$ for all 


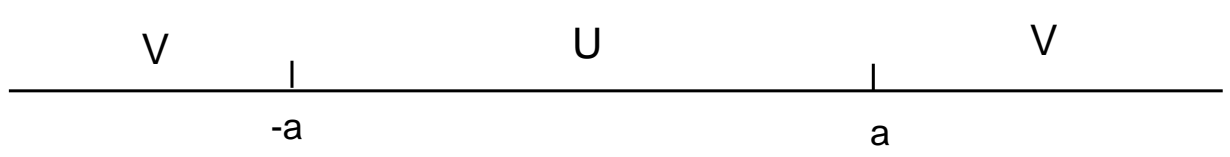

Figure 2

$0 \leq \epsilon \leq \epsilon_{0}$. Therefore, there is a constant $K>0$ such that $|\log | g_{i}^{\prime}(x)|| \leq K$ for all $x \in[-1,0]$, for $i=0$ or 1 , and for all $0 \leq \epsilon \leq \epsilon_{0}$.

Let

$$
U(\epsilon)=\left[-a_{\epsilon}, a_{\epsilon}\right]
$$

and let

$$
V(\varepsilon)=\left[-1,-a_{\epsilon}\right] \cup\left[a_{\epsilon}, 1\right]
$$

(See Figure 2.)

Dividing the interval $[-1,1]$ into two parts $U(\epsilon)$ and $V(\epsilon)$ is one of the key points in the proof: on $V(\epsilon), f_{\epsilon}$ is $C^{3}$ and expanding, one can apply the naive distortion lemma (see Lemma 1), and on $U(\epsilon)$, although the derivative of $f_{\epsilon}$ may be zero, but Theorem 2 takes care of it (see Lemma 2). We also note that $f_{\epsilon}(U(\epsilon))=[0,1+\epsilon]$ and $f_{\epsilon}(V(\epsilon))=[-1,0]$.

From (c) of Definition 3, there is a constant $0<\mu_{0}<1$, which is independent of $\epsilon$, such that

$$
\left|g_{0, \epsilon}^{\prime}(-1)\right|,\left|g_{1, \epsilon}^{\prime}(-1)\right| \leq \mu_{0} .
$$

By considering $T=[-1,1+\epsilon]$ and $x=0$ and applying the inequality $(*)$, we have that

$$
\left|g_{0, \epsilon}^{\prime}(0)\right|,\left|g_{1, \epsilon}^{\prime}(0)\right| \leq \frac{1}{2}
$$

Then applying the inequality $(* *)$, we get

$$
\left|g_{0, \epsilon}^{\prime}(x)\right|,\left|g_{1, \epsilon}^{\prime}(x)\right| \leq \mu=\min \left\{\mu_{0}, \frac{1}{2}\right\}<1
$$

for all $x \in[-1,0]$ and all $0 \leq \epsilon \leq \epsilon_{0}$.

Let $w_{m}=j_{0} j_{1} \ldots j_{m-1}$ be a sequence of 0's and 1's, let $g_{w_{m}}=g_{j_{0}} \circ \cdots \circ g_{j_{m-1}}$, and let $I_{w_{m}}=g_{w_{m}}([-1,1])$. Let $w_{n}=i_{0} \ldots i_{n-1}$ be another sequence of 0 's and 1 's and let $g_{w_{n}}=g_{i_{0}} \circ \ldots \circ g_{i_{n-1}}$. Let $w_{n+m}=w_{n} w_{m}=i_{0} \ldots i_{n-1} j_{0} \ldots j_{m-1}$ and let $g_{w_{n+m}}=g_{w_{n}} \circ g_{w_{m}}$. Then $I_{w_{n+m}}=g_{w_{n+m}}([-1,1])=g_{w_{n}}\left(I_{w_{m}}\right)$. Let $w_{k+m}=i_{n-k} \ldots i_{n-1} w_{m}$ and $I_{w_{k+m}}=g_{w_{k+m}}([-1,1])$ for $k=1, \ldots, n$.

Lemma 1. There is a constant $K>0$ independent of $\epsilon$ such that if $I_{w_{k+m}} \subseteq V(\epsilon)$ for $k=1, \ldots, n$, then

$$
\left|\log \left(\frac{\left|g_{w_{n}}^{\prime}(x)\right|}{\left|g_{w_{n}}^{\prime}(y)\right|}\right)\right| \leq K
$$

for all $x$ and $y$ in $I_{w_{m}}$ and all $0 \leq \epsilon \leq \epsilon_{0}$. 
Proof. Let $w_{k}=i_{n-k} \ldots i_{n-1}$, let $g_{w_{k}}=g_{i_{n-k}} \circ \ldots \circ g_{i_{n-1}}$, and let $x_{k}=g_{w_{k}}(x)$ and $y_{k}=g_{w_{k}}(y)$ for $k=1, \ldots, n$. Set $x_{0}=x$ and $y_{0}=y$. Since we have

$$
\frac{\left|g_{w_{n}}^{\prime}(x)\right|}{\left|g_{w_{n}}^{\prime}(y)\right|}=\prod_{k=0}^{n-1} \frac{\left|g_{i_{n-k-1}}^{\prime}\left(x_{k}\right)\right|}{\left|g_{i_{n-k-1}}^{\prime}\left(y_{k}\right)\right|}
$$

then

$$
\mathcal{A}=\left|\log \left(\frac{\left|g_{w_{n}}^{\prime}(x)\right|}{\left|g_{w_{n}}^{\prime}(y)\right|}\right)\right| \leq \sum_{k=0}^{n-1}|\log | g_{i_{n-k-1}}^{\prime}\left(x_{k}\right)|-\log | g_{i_{n-k-1}}^{\prime}\left(y_{k}\right)|| .
$$

Since $\left|\left(|\log | g_{i}^{\prime}(x) \mid\right)^{\prime}\right| \leq K$ for all $i=0$ and 1 , all $x \in[-1,0]$, and all $0 \leq \epsilon \leq \epsilon_{0}$ and since $x_{k}$ and $y_{k}$ are in $[-1,0]$ for all $k=0, \ldots, n-1$, there is a constant $K>0$ independent of $\epsilon$ such that

$$
\mathcal{A} \leq K \sum_{k=0}^{n-1}\left|x_{k}-y_{k}\right|
$$

Because $g_{0}$ and $g_{1}$ restricted to $[-1,0]$ are contracting and because $I_{w_{k+m}} \subseteq[-1,0]$ for $0 \leq k \leq n-1$, we have

$$
\sum_{k=0}^{n-1}\left|x_{k}-y_{k}\right| \leq \sum_{k=0}^{n-1} \mu^{k} \leq \frac{1}{1-\mu}
$$

Thus

$$
\mathcal{A} \leq \frac{K}{1-\mu} .
$$

Here $K /(1-\mu)$ is the constant we want.

Lemma 2. There is a constant $K>0$ independent of $\epsilon$ such that if $I_{w_{m}} \subseteq U(\epsilon)$, then

$$
\left|\log \left(\frac{\left|g_{w_{n}}^{\prime}(x)\right|}{\left|g_{w_{n}}^{\prime}(y)\right|}\right)\right| \leq K
$$

for all $x$ and $y$ in $I_{w_{m}}$ and for all $0 \leq \epsilon \leq \epsilon_{0}$.

Proof. The distance $\operatorname{dist}(U(\epsilon), \partial[-1,1+\epsilon])=1+\epsilon-a_{\epsilon}$ exceeds a positive constant $K$ for all $0 \leq \epsilon \leq \epsilon_{0}$. Since $g_{w_{n}}$ is a $C^{3}$-diffeomorphism defined on $(-1,1+\epsilon)$ having $S\left(g_{w_{n}}\right) \geq 0$, Corollary 1 tells us that

$$
\left|\log \left(\frac{\left|g_{w_{n}}^{\prime}(x)\right|}{\left|g_{w_{n}}^{\prime}(y)\right|}\right)\right|=\left|\int_{x}^{y} N\left(g_{w_{n}}\right)(\xi) d \xi\right| \leq \frac{2|x-y|}{K} .
$$

This implies the lemma.

Remark 4. The sequence of intervals $\left\{I_{w_{k+m}}\right\}_{k=0}^{n}$ may enter the critical region $U$ many times. However, Lemma 2 says that the nonlinearity of $g_{w_{n}}$ on $I_{w_{m}}$ can be determined just by looking at the position of the first interval $I_{w_{m}}$. It looks like magic but this magic is caused by a strong condition that $g_{w_{n}}$ is $C^{3}$ and the Schwarzian derivative of $g_{w_{n}}$ is bounded below by 0 . This condition is similar to the analytic condition in complex analysis. In that context, we have Koebe's theorem (see [BI]) showing a similar magic fact. A more detailed analysis of this magic phenomenon under the $C^{1+\alpha}$ condition is given in [JI3], [JI4]. There is an intuitive reason about why Lemma 2 is true: let $P S O=\{-1,1\}$ be the post-critical orbit of $f$ and let dist mean the distance. Since $I_{w_{m}}$ falls in the critical region $U$, the ratio 
$\left|I_{w_{m}}\right| / \operatorname{dist}\left(I_{w_{m}}, P S O\right)$ is bounded. Now we can look at the next interval, say $I_{w_{k+w}}$, which falls in $U$. Since $I_{w_{k+m}}$ and 0 are the preimage of $I_{w_{m}}$ and $P S O$ under some iterate of $f$ and since $f \mid V$ is $C^{3}$ and expanding, we get that $\left|I_{w_{k+m}}\right| / \operatorname{dist}\left(I_{w_{k+m}},\{0\}\right)$ is also bounded by Lemma 1 . Similarly one can examine all intervals which fall in $U$ and consider the ratios of the lengths of these intervals and the distances between them and 0 . One can prove that any of these ratios is exponentially smaller than the previous one. The total nonlinearity of $g_{w_{n}}$ relates to the summation of these ratios. Therefore, it is bounded.

Proof of Theorem 1. For any interval $I_{w_{n}}=g_{w_{n}}([-1,1])$, we have

$$
I_{w_{n}}=I_{w_{n} 0} \cup G_{w_{n}} \cup I_{w_{n} 1},
$$

which is (i) of Definition 1. Let

$$
g g_{w_{n} 0}(\epsilon)=\frac{\left|G_{w_{n}}\right|}{\left|I_{w_{n} 0}\right|} \quad \text { and } \quad g g_{w_{n} 1}(\epsilon)=\frac{\left|G_{w_{n}}\right|}{\left|I_{w_{n} 1}\right|}
$$

and let

$$
b r_{w_{n} 0}(\epsilon)=\frac{\left|I_{w_{n} 0}\right|}{\left|I_{w_{n}}\right|} \quad \text { and } \quad b r_{w_{n} 1}(\epsilon)=\frac{\left|I_{w_{n} 1}\right|}{\left|I_{w_{n}}\right|} .
$$

Let $l g=l g(\epsilon)$ be the size of the leading gap $G_{*}$. From the argument after Definition 3 , there is a constant $K>0$ such that

$$
K^{-1} \leq \frac{\lg (\epsilon)}{\epsilon^{\frac{1}{\gamma}}} \leq K
$$

for all $0<\epsilon \leq \epsilon_{0}$. Moreover, there is a constant $K>0$ such that

$$
K^{-1} \leq \frac{g g_{w_{1}}(\epsilon)}{\epsilon^{\frac{1}{\gamma}}} \leq K
$$

and such that

$$
K^{-1} \leq b r_{w_{1}}(\epsilon) \leq K
$$

for all $0 \leq \epsilon \leq \epsilon_{0}$ and for $w_{1}=0$ or 1 ,

Let $w_{n}=i_{0} \ldots i_{n-1}$ be any sequence of 0's and 1's of length $n>1$. Let $w_{k}=$ $i_{n-k} \ldots i_{n-1}$, let $g_{w_{k}}=g_{i_{n-k}} \circ \cdots \circ g_{i_{n-1}}$, and let $I_{k}=g_{w_{k}}([-1,1])$ for $k=1, \ldots$, $n$. The sequence of intervals $\left\{I_{k}\right\}_{k=1}^{n}$ satisfies either

(I) $I_{k} \subseteq V(\epsilon)$ for all $2 \leq k \leq n$ or

(II) there is an integer $2 \leq m \leq n$ such that $I_{k} \subseteq V(\epsilon)$ for all $2 \leq k<m$ and such that $I_{m} \subseteq U(\epsilon)$.

If the sequence $\left\{I_{k}\right\}_{k=1}^{n}$ satisfies (I), then we apply Lemma 1 and the inequalities $\left.{ }^{* * *}\right)$ and $\left(^{* * *}\right)$ to get a constant $K>0$ independent of $\epsilon$ such that

$$
K^{-1} \leq \frac{g g_{w_{n} i}(\epsilon)}{\epsilon^{\frac{1}{\gamma}}} \leq K
$$

and such that

$$
K^{-1} \leq b r_{w_{n} i}(\varepsilon) \leq K
$$

for all $0<\epsilon \leq \epsilon_{0}$ and $i=0$ and 1 . 
If the sequence $\left\{I_{k}\right\}_{k=1}^{n}$ satisfies (II), we have that $I_{m} \subseteq U(\epsilon)$ and $I_{k} \subseteq V(\epsilon)$ for all $2 \leq k<m$. Following the argument in (I), we also have that

$$
K^{-1} \leq \frac{g g_{w_{m-1} i}(\epsilon)}{\epsilon^{\frac{1}{\gamma}}} \leq K
$$

and that

$$
K^{-1} \leq b r_{w_{m-1} i}(\varepsilon) \leq K
$$

for all $0<\epsilon \leq \epsilon_{0}$ and $i=0$ and 1 .

From (b) of Definition $3, f_{\epsilon}(x) \mid I_{m}$ is comparable with

$$
x \mapsto(1+\epsilon)-(2+\epsilon)|x|^{\gamma}
$$

for all $0<\epsilon \leq \epsilon_{0}$. There is thus a constant $K>0$ independent of $\epsilon$ such that

$$
K^{-1} \leq \frac{g g_{w_{m} i}(\epsilon)}{g g_{w_{m-1} i}} \leq K
$$

and such that

$$
K^{-1} \leq \frac{b r_{w_{m} i}(\varepsilon)}{b r_{w_{m-1} i}(\varepsilon)} \leq K
$$

for all $0<\epsilon \leq \epsilon_{0}$ and $i=0$ and 1 .

From Lemma 2, there is a constant $K>0$ independent of $\epsilon$ such that

$$
K^{-1} \leq \frac{g g_{w_{n} i}(\epsilon)}{g g_{w_{m} i}(\epsilon)} \leq K
$$

and such that

$$
K^{-1} \leq \frac{b r_{w_{n} i}(\varepsilon)}{b r_{w_{m} i}(\varepsilon)} \leq K
$$

for all $0<\epsilon \leq \epsilon_{0}$ and $i=0$ and 1 .

By combining the above estimates, we get that in both (I) and (II), there is a constant $K>0$ independent of $\epsilon$ such that

$$
K^{-1} \leq \frac{g g_{w_{n} i}(\epsilon)}{\epsilon^{\frac{1}{\gamma}}} \leq K
$$

and such that

$$
K^{-1} \leq b r_{w_{n} i}(\varepsilon) \leq K
$$

for all $0<\epsilon \leq \epsilon_{0}$ and $i=0$ and 1 (refer to Remark 4).

For each fixed $\epsilon>0$, since $I_{w_{n}}=I_{w_{n} 0} \cup G_{w_{n}} \cup I_{w_{n} 1}$, we can further get constants $0<\nu<1$ and $K>0$ such that

$$
\left|I_{w_{n}}\right| \leq K \nu^{n}
$$

for all $I_{w_{n}} \in \mathcal{I}_{n}$ and all $0 \leq n<\infty$. This implies that

$$
C S_{\epsilon}=\bigcap_{n=0}^{\infty} \bigcup_{I \in \mathcal{I}_{n}} I
$$

contains no interval. Therefore it is totally disconnected, and furthermore, a Cantor set. 


\section{REFERENCES}

[AH] L. Ahlfors, Lectures on Quasiconformal Mappings, Van Nostrand (1966). MR 34:336

[AR] V. I. Arnold, Ordinary Differential Equations, M.I.T. Press: Cambridge, MA (1973 (Russian original, Moscow, 1971)). MR 50:13679

[BI] L. Bieberbach, Conformal Mapping, Chelsea Publishing Company, New York, 1953. MR 14:462c

[CT] P. Coullet and C. Tresser, Itération d'endomorphismes et groupe de renormalisation, C. R. Acad. Sci. Paris Ser., A-B 287 (1978), A577-A580. MR 80b:58043

[CE] P. Collet and P. Eckmann, Iterated Maps on the Interval as Dynamical Systems, Progress in Physics 1 (1980). MR 82j:58078

[FE1] M. Feigenbaum, Quantitative universality for a class of non-linear transformations, J. Stat. Phys. 19 (1978), 25-52. MR 58:18601

[FE2] M. Feigenbaum, The universal metric properties of non-linear transformations, J. Stat. Phys. 21 (1979), 669-706. MR 82e:58072

[JI1] Y. Jiang, Geometry of geometrically finite one-dimensional maps, Commun. in Math. Phys. 156 (1993), 639-647. MR 95f:58033

[JI2] Y. Jiang, Generalized Ulam-von Neumann transformations, Thesis, Graduate School of CUNY (1990).

[JI3] Y. Jiang, Nonlinearity, quasisymmetry, differentiability, and rigidity in one-dimensional dynamics, Preprint.

[JI4] Y. Jiang, Dynamics of certain non-conformal semi-groups, Complex Variables 22 (1993), 27-34. MR 95g:30025

[LA1] O. E. Lanford III, A computer-assistant proof of the Feigenbaum conjecture, Bull. Amer. Math. Soc. 6 (1982), 427-434. MR 83g:58051

[LA2] O. E. Lanford III, A shorter proof of the existence of Feigenbaum fixed point, Commun. in Math. Phys. 96 (1984), 521-538. MR 86c:58121

[MC] C. McMullen, Renormalization and 3-manifolds which fiber over the circle, Preprint.

[MV] W. de Melo and S. van Strien, One-Dimensional Dynamics, Springer-Verlag, Berlin, Heidelberg, 1993. MR 95a:58035

[SM] S. Smale, The Mathematics of Time: Essays on Dynamical Systems, Economic Processes and Related Topics, Springer-Verlag, New York, Heidelberg, Berlin, 1980. MR 83a:01068

[SU] D. Sullivan, Bounds, quadratic differentials, and renormalization conjectures, Mathematics into the Twenty-First Century 2 (1992). MR 93k:58194

Department of Mathematics, Queens College, The City University of New York, Flushing, New York 11367-1597 and Department of Mathematics, Graduate School of The City University of New York, New York, New York 10036

E-mail address: yunqc@yunping.math.qc.edu 